\title{
The Gamma-Ray Background from Blazars: A New Look
}

\author{
F.W. Stecker ${ }^{1}$ \\ Laboratory for High Energy Astrophysics \\ NASA Goddard Space Flight Center, Greenbelt, MD 20771 \\ and \\ M.H. Salamon \\ High Energy Astrophysics Institute, Physics Department \\ University of Utah, Salt Lake City, UT 84112 \\ ${ }^{1}$ also, High Energy Astrophysics Institute, Physics Department \\ University of Utah, Salt Lake City, UT 84112
}

\begin{abstract}
We present the results of a new model calculation of the $\gamma$-ray background produced by unresolved blazars, using the second EGRET catalogue and taking account of flaring. These results are compared to the preliminary $\gamma$-ray background spectrum reported recently by the EGRET team. We find that blazars can account for the entire extragalactic $\gamma$-ray background observed by EGRET. In addition the EGRET spectrum shows some evidence of a signature for the blazar background, a concavity in the spectrum first pointed out in our earlier paper.
\end{abstract}

Subject headings: galaxies: active - gamma-rays: theory - quasars: general

\section{Introduction}

An isotropic, thus presumably extragalactic, component of the cosmic $\gamma$-ray background above $30 \mathrm{MeV}$ was first detected by the SAS-2 satellite (Fichtel, Simpson, and Thompson, 1978, Thompson and Fichtel, 1982) and has more recently been measured by the EGRET experiment on the Compton Gamma Ray Observatory (Fichtel, 1995). The EGRET team has also discovered that the only significant extragalactic point sources which produce $\gamma$-rays in this energy range are the catagory of objects called blazars (Thompson et al., 1995, Dermer and Gehrels, 1995). These objects are active galactic nuclei whose jets are nearly pointing 
along our line of sight, resulting in enhanced luminosity and variability within a narrow cone around the jet axis, due to the highly relativistic motion of the source region within the jet. It can also be shown that physical mechanisms for producing diffuse radiation, such as the annihilation of antimatter or of supersymmetry dark matter particles, cannot produce an isotropic $\gamma$-ray flux above a few hundred $\mathrm{MeV}$ of the magnitude observed (Stecker, 1989). Blazars would thus appear to be the only present candidates for producing the observed extragalactic $\gamma$-radiation in this energy range.

With the new measurement of the extragalactic $\gamma$-ray radiation background (EGRB) by EGRET (Fichtel, 1995), and an enhanced blazar data set in the second EGRET catalogue (Thompson et al., 1995), we have been led to reconsider an earlier model of $\gamma$-ray background production by blazars (Stecker, Salamon, and Malkan, 1993, Salamon and Stecker, 1994), which we have now expanded to include the effects of both flux and spectral variability of blazars due to flaring. A particularly important observation by EGRET is the apparent hardening of spectra of blazars when they are in a flare state, which plays a key role in our analysis. Although this model is simplistic, it provides an excellent fit to both the shape and amplitude of the EGRB, while simultaneously accounting for both the number of blazars seen by EGRET, and their observed flux distribution.

\section{The Basic Model}

Our earlier model of $\gamma$-ray background production by blazars (Stecker, Salamon, and Malkan, 1993, Salamon and Stecker, 1994) made the basic assumption that blazars seen in $\gamma$-rays above $100 \mathrm{MeV}$ are also seen in the radio $(2.7$ to $5.0 \mathrm{GHz}$ ) as flat spectrum radio quasars (FSRQs), with fewer blazars seen than FSRQs due to the existence of a narrower beaming angle in $\gamma$-rays than in the radio (see also Dermer, 1995). With the additional assumption that the radio and $\gamma$-ray luminosity of these objects are linearly related (see also Padovani, 1993), one is able to use pre-existing radio luminosity functions (RLF) to estimate the blazar $\gamma$-ray luminosity function (GLF), which can then be used to calculate the contribution of unresolved blazars to the isotropic $\gamma$-ray background. A constraint on the model is that the number of observed blazars in $\gamma$-rays estimated with this GLF correspond to the number actually observed by EGRET, given their instrumental flux sensitivity.

We use here the same RLF as in our earlier work (Dunlop and Peacock, 1990),

$$
\rho_{r}\left(P_{r}, z\right)=10^{-8.15}\left\{\left[\frac{P_{r}}{P_{c}(z)}\right]^{0.83}+\left[\frac{P_{r}}{P_{c}(z)}\right]^{1.96}\right\}^{-1}
$$

where $\log _{10} P_{c}(z)=25.26+1.18 z-0.28 z^{2}$, and the units of source luminosity $P_{r}$ and co- 
moving density $\rho_{r}$ are, respectively, $\mathrm{W} \mathrm{Hz}^{-1} \mathrm{sr}^{-1}$ and $\mathrm{Mpc}^{-3}$ (unit interval of $\left.\log _{10} P\right)^{-1}$. $P_{r}$ is the differential radio luminosity (per steradian) measured at $2.7 \mathrm{GHz}$; the RLF is constructed assuming pure luminosity evolution, with $\Omega_{0}=1.0$ and $H_{0}=50 \mathrm{~km} \mathrm{~s}^{-1} \mathrm{Mpc}^{-1}$.

The GLF is then given by

$$
\rho_{\gamma}\left(P_{\gamma f}, z\right)=\eta \rho_{r}\left(P_{\gamma f} / \kappa, z\right)
$$

where $\eta=\left(\theta_{\gamma} / \theta_{r}\right)^{2}$ is the reduction of the number of $\gamma$-ray blazars compared to FSRQs by virtue of a hypothesized smaller beaming angle $\theta_{\gamma}$, and $\kappa$ is the proportionality constant between the differential luminosities, $P_{\gamma f}=\kappa P_{r}$, which we evaluate at the fiducial energies $E_{f}=100 \mathrm{MeV}$ and $\nu_{f}=2.7 \mathrm{GHz}$. The parameter $\kappa$ is estimated from the distribution of ratios of measured $\gamma$-ray and radio fluxes of the EGRET blazars, where radio fluxes at 2.7 GHz were obtained from Wall and Peacock, 1985, Kühr et al., 1981, and Ledden and O'DeII, 1985 .

With this GLF, and appropriate values for $\eta$ and $\kappa$, an estimate of the contribution to the EGRB from blazars was made (Stecker, Salamon, and Malkan, 1993, Salamon and Stecker, 1994) and found to be a significant fraction of the measured EGRB. In particular, we noted that the spectrum of the EGRB due to blazars would be softer at lower energies, and harder at higher energies, a feature which appears to be present in the most recent EGRB measurement (Eichtel, 1995). We also note that a recent analysis using EGRET data to obtain an estimate of the GLF at the high end of the blazar luminosity distribution is consistent with a luminosity and redshift dependence of source distribution given by Eq.11 (Chiang et al., 1995).

An obvious oversimplification of the earlier model was its lack of accounting for the wellknown time variability of blazars, a complex phenomenon which does not lead itself easily to simple characterizations. It is known that blazars can vary significantly over timescales of the order of a couple of days or less (Jang and Miller, 1995, Wagner and Witzel, 1995). Significant flaring in the $\mathrm{GeV} \gamma$-ray range is perhaps best illustrated by the intense flare which occurred during June 1991 in the source 3C279 (Kniffen et al., 1993). Such flaring, in $\gamma$ rays as well as in other regions of the electromagnetic spectrum, appears to be associated with shocks propagating down relativistic jets and with the subsequent acceleration of relativistic particles by these shocks (Jang and Miller, 1995, Valtaoja and Teräsranta, 1995).

In this paper we incorporate this phenomenon in a simple way, postulating the existence of two "states" of a blazar, a "quiescent" state and a "flaring" state, where the quiescent blazar luminosity is amplified by a fixed factor $A$ during a flare. It is assumed that a blazar spends a fraction $\zeta$ of its time in a flare state, where $\zeta \ll 1$.

In addition, the distribution of spectral indices of flare-state blazars is taken to be harder 


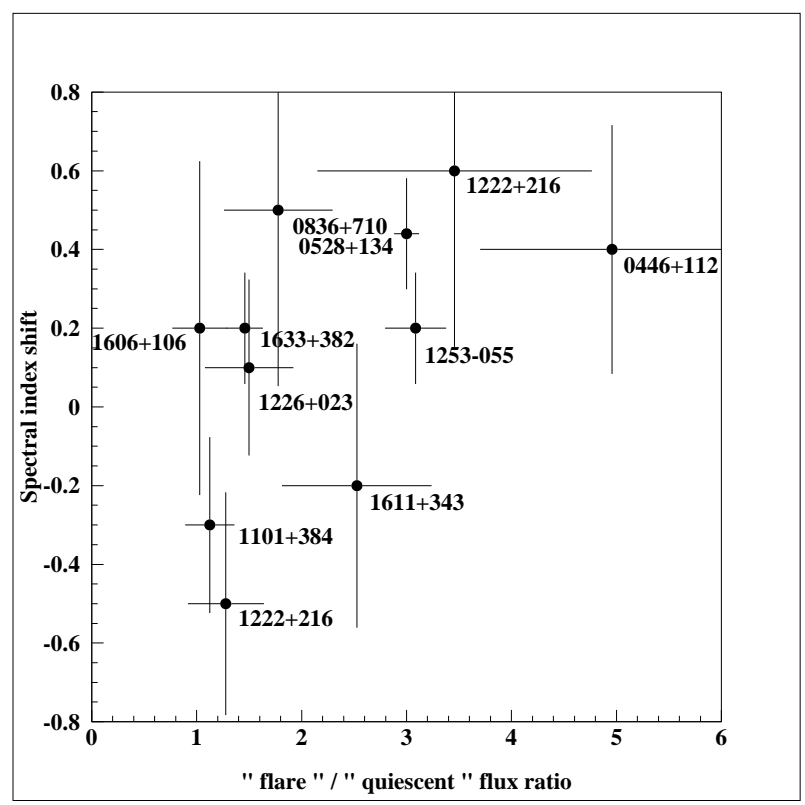

Fig. 1.- A plot of the shift in measured blazar spectral index versus flux ratio for various blazars observed by ERGET during different viewing periods. The flux values used in the ratios were those obtained for the viewing periods in which spectral indices are determined. The datum for $0528+134$ is taken from Mukherjee et al.(1995).

than that of quiescent-state blazars (Sreekumar et al., 1995). This is supported by EGRET's observations of flares in which the hardening of the spectral index is statistically significant e.g., 0528+134 (Mukherjee et al., 1995), and also as a statistical trend as seen in Figure 1. Here we have used the $2^{\text {nd }}$ EGRET catalogue (Thompson et al., 1995) and considered all blazars for which more than one spectral index is given (corresponding to different viewing periods). For each object, the ratio of the integral $\gamma$-ray fluxes of the two different periods is plotted against the spectral index shift, where we construct the flux ratio so that it is always greater than unity. A suggestive trend is apparent, indicating a typical hardening of the spectral index by $\sim 0.3$ during strong $(A>3)$ flares.

The spectral index $\alpha$ is defined by $E \frac{d F}{d E}(E) \propto E^{-\alpha}$, where $\frac{d F}{d E}(E)$ is the differential number flux of $\gamma$-rays at energy $E$; equivalently, the $\gamma$-ray differential luminosity at the source varies with $\gamma$-ray energy as $P_{\gamma}(E)=P_{\gamma f}\left(E / E_{f}\right)^{-\alpha}$, consistent with all observed blazar $\gamma$-ray spectra. The blazars' distribution of spectral indices $\alpha$ is determined from the $2^{\text {nd }}$ EGRET catalogue,

$$
p(\alpha)=\frac{1}{N} \sum_{i=1}^{N} \frac{1}{\sigma_{i} \sqrt{2 \pi}} e^{-\left(\alpha-\alpha_{i}\right)^{2} / 2 \sigma_{i}^{2}},
$$

where $\alpha_{i}$ and $\sigma_{i}$ are the catalogue's spectral index and error for the $i^{\text {th }}$ of $\mathrm{N}$ blazars. We 
simply shift this distribution to obtain the separate distributions for the flare and quiescent states: $p^{f}(\alpha)=p\left(\alpha-\Delta \alpha_{f}\right)$, and $p^{q}(\alpha)=p\left(\alpha-\Delta \alpha_{q}\right)$, where we take $\Delta \alpha_{q}=0.20$ and $\Delta \alpha_{f}=-0.05$, as discussed below. As there is no evidence for a change in the blazar spectral index distribution with redshift $z$ in the EGRET catalogue, we assume none.

Our GLFs for the two states of blazars, quiescent and flaring, is a modification of Eq.2,

$$
\rho_{\gamma}\left(P_{\gamma f}, z\right)=(1-\zeta) \eta \rho_{r}\left(\frac{P_{\gamma f}}{\kappa}, z\right)+\zeta \eta \rho_{r}\left(\frac{P_{\gamma f}}{A \kappa}, z\right)
$$

where the quiescent and flare $\gamma$-ray luminosities, $P_{\gamma}^{q}$ and $P_{\gamma}^{f}$, are related to the radio luminosity as $P_{\gamma}^{q}=\kappa P_{r}$ and $P_{\gamma}^{f}=A \kappa P_{r}$. We note that although variability is seen in the radio at higher frequencies, very little is observed at $2.7 \mathrm{GHz}$, the frequency at which our chosen RLF is determined (Reich, et al., 1993, Valtaoja and Teräsranta, 1995). Thus we need not be concerned with the effects of variability in our reference RLF.

\section{The Observed Flux Distribution}

For a blazar at redshift $z$ with luminosity $P_{\gamma f}$ (which is the luminosity evaluated at the fiducial energy $E_{f}$ at the source) one can show that the integral flux $F\left(E_{f}\right)$ at Earth is given by

$$
F\left(E_{f}\right)=P_{\gamma f} /\left[\alpha(1+z)^{\alpha+1} r^{2} R_{0}^{2}\right],
$$

where $r$ is the co-moving coordinate of the blazar, and $R_{0}$ is the present cosmological scale factor, $R_{0} r=\left(2 c / H_{0}\right)\left[1-(1+z)^{-1 / 2}\right]$ for $\Omega_{0}=1$ and $\Lambda=0$. (The absence of $4 \pi$ in the denominator is because $P_{\gamma f}$ is a differential luminosity per steradian.)

The number of sources at redshift $z$ seen at Earth with an integral flux $F$ is given by

$$
\frac{d N}{d F d z} \Delta z \Delta F=4 \pi R_{0}^{3} r^{2} \Delta r \rho_{\gamma}\left(P_{\gamma f}, z\right) \Delta\left(\log _{10} P_{\gamma f}\right)
$$

where the relation between $F$ and $P_{\gamma f}$ is fixed by Eq.5. With this, the number of sources present per unit logarithm of flux $F$ is found by integrating Eq.6 over redshift,

$$
\frac{d N}{d\left(\log _{10} F\right)}=\frac{16 \pi c^{3}}{H_{0}^{3}} \int_{0}^{z_{\max }} d z \frac{\left[(1+z)^{1 / 2}-1\right]^{2}}{(1+z)^{5 / 2}}\left\{\eta(1-\zeta) \rho_{r}\left[\frac{P_{\gamma f}}{\kappa}, z\right]+\eta \zeta \rho_{r}\left[\frac{P_{\gamma f}}{A \kappa}, z\right]\right\}
$$

where we take $z_{\max }=5$.

For the choice of parameters discussed in Sec. 4 below, the number of blazars in the sky as a function of integrated flux at Earth is shown in Figure 2. Here we show the number 
seen as quiescent-state blazars, and the number seen as flare-state blazars, and their total. Note that the two separate contributions represent the same underlying (radio) distribution, shifted along the abscissa by the flare factor $A(A=5)$, and along the ordinate by the flare duty cycle, $\zeta(\zeta=0.03)$. Also shown on the figure are the number of blazars seen by EGRET as a function of flux $F\left(E_{f}=100 \mathrm{MeV}\right)$, binned logarithmically. We take as our sample the 50 blazars identified in the 2nd EGRET catalogue, along with 14 unidentified EGRET sources above 30 degrees galactic latitude which are most likely to be blazars (Mukherjee et al. 1995). The agreement is seen to be quite good, both in the shape and amplitude of the experimental distribution. The dropoff of data below $F=2 \times 10^{-7} \mathrm{~cm}^{-2} \mathrm{~s}^{-1}$ is due to the loss of blazar detection efficiency near EGRET's minimum flux sensitivity of $\sim 1 \times 10^{-7}$ $\mathrm{cm}^{-2}-\mathrm{s}^{-1}$. In addition, there is incompleteness in the sky survey at this sensitivity near the galactic plane, owing to the enhanced galactic foreground emission.

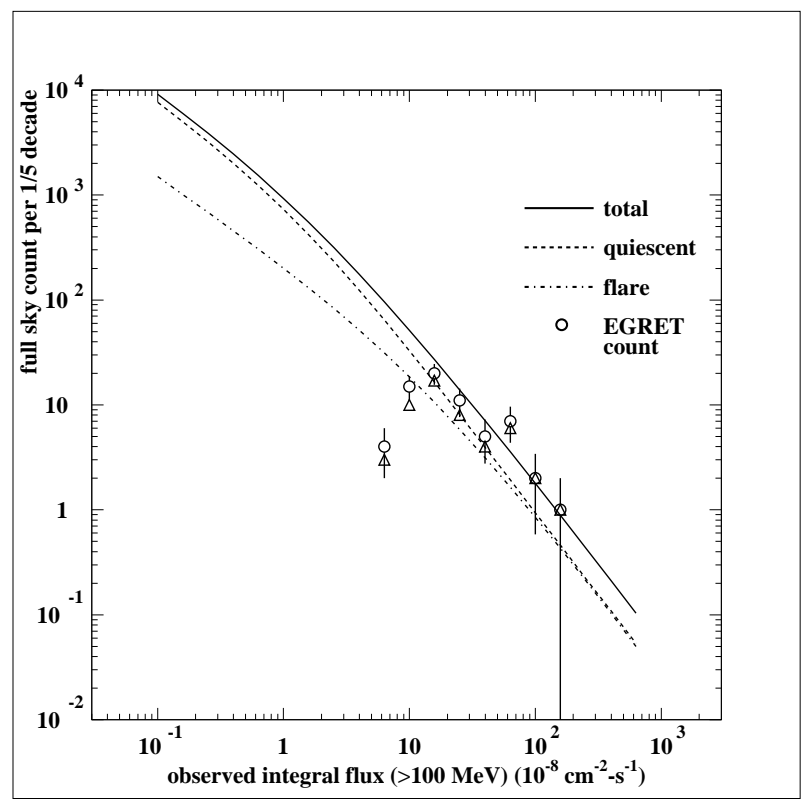

Fig. 2.- The the predicted distibution of sources at various flux levels using the model parameters described in the text. The open circles and their statistical error bars represent the 50 objects identified as blazars in the second EGRET catalogue, plus the 14 unidentified, high-latitude sources which are presumably blazars as well. The open triangles (no error bars) show only the 50 identified blazars' count per logarithmic bin. Note that the ordinate is the full sky count per one-fifth decade of flux.

The curves in Figure 2 give the number of sources vs. flux predicted at a single point in time. At any one point in time, a certain fraction of the observed blazars will be in a flare state. At another point in time, however, a different set of blazars will be in a flare 
state. Since EGRET has viewed the entire sky multiple times (although not uniformly after its inital full sky map), one expects that the number of flaring blazars seen by EGRET would be greater than that indicated by the flare curve of Figure 2. How much this should increase the contribution of the flare component to the total EGRET blazar count is not clear, as many of the flaring blazars are also detected in the quiescent state (von Montigny et al., 1995). Complicating this issue is the fact that many of the multiple observations are made significantly off EGRET's vertical axis, increasing the minimum flux sensitivity for detection. The mean number of observations of an object (with and without detections) is $\sim 7$, at various levels of sensitivity. Were we to increase the flare contribution to the total EGRET count by a factor of 3 to account for multiple viewings, we would still be reasonably consistent with the data (allowing for off-axis inefficiencies at the lower flux levels).

An important point is that the distribution of spectral indices $\alpha$ measured by EGRET contains those from both the quiescent and flare state populations, with the flare states contributing heavily to the EGRET distribution, as Figure 2 and the above discussion indicates. Therefore the quiescent state population, which we show below dominates the extragalactic $\gamma$-ray background radiation, has a softer distribution of spectral indices than that seen by EGRET.

\section{The Extragalactic Gamma-Ray background}

Unresolved blazars, i.e., those which are below detection threshold, will contribute to a diffuse, isotropic background of $\gamma$-ray radiation. We now consider whether unresolved blazars may be responsible for the entire observed extragalactic $\gamma$-ray background.

The differential number flux $\frac{d F}{d E}{ }^{(1)}$ of $\gamma$-rays received at Earth from a single source at redshift $z$ is given by Salamon and Stecker, 1994

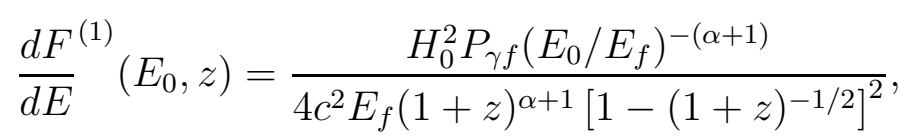

where $E_{0}$ is the $\gamma$-ray energy at Earth, $E_{f}$ is the fiducial energy $(100 \mathrm{MeV}$ ), and $\alpha$ is the spectral index of the single source. We note that we neglect the minor effect of $\gamma$-ray extinction off the IR-to-UV diffuse background (Stecker, deJager, and Salamon, 1992) for our energy region of interest, 0.1 to $100 \mathrm{GeV}$.

The total differential flux from unresolved blazars at a redshift $z$ is found by integrating Eq.8 over the GLF $\rho_{\gamma}\left(P_{\gamma f}, z\right)$ :

$$
\frac{d F}{d E}\left(E_{0}\right)=4 \pi R_{0}^{3} r^{2} d r \int d \alpha p(\alpha) \int_{P_{\gamma f, \min }}^{P_{\gamma f, \max }} \frac{d F^{(1)}}{d E}\left(E_{0}, z\right) \rho_{\gamma}\left(P_{\gamma f}, z\right) d\left(\log _{10} P_{\gamma f}\right)
$$


where $P_{\gamma f, \max }$ is the luminosity of a blazar at redshift $z$ which is just below the threshold for detection (found by subtituting EGRET's integral flux sensitivity $F_{\min }^{\text {EGET }}(100 \mathrm{MeV})$ into Eq. (5), and an integration over the blazar spectral index distribution is made.

We next integrate over redshift to obtain the total diffential flux at Earth at energy $E_{0}$. We substitute Eq.⿴囗十 into Eq.9, expressing the luminosity integral as one over the RLF (Eq.11), obtaining

$$
\begin{aligned}
\frac{d F}{d E}\left(E_{0}\right)= & \frac{c}{H_{0} E_{f} \ln 10}\left\{\int p^{q}(\alpha)\left(\frac{E_{0}}{E_{f}}\right)^{(\alpha+1)} \int_{0}^{z_{\max }} \frac{d z}{(1+z)^{\alpha+5 / 2}} \int_{P_{r, \min }^{P_{m}}}^{q}(1-\zeta) \eta \rho\left(P_{r}, z\right) d P_{r}\right. \\
& \left.+\int p^{f}(\alpha)\left(\frac{E_{0}}{E_{f}}\right)^{(\alpha+1)} \int_{0}^{z_{\max }} \frac{d z}{(1+z)^{\alpha+5 / 2}} \int_{P_{r, \min }^{f}}^{P_{\max }(z)} \zeta \eta \rho\left(P_{r}, z\right) d P_{r}\right\} .
\end{aligned}
$$

The radio luminosity limits are given by $P_{r, \text { max }}^{q}(z)=P_{\gamma f, \max }(z) / \kappa$, and $P_{r, \text { max }}^{f}(z)=P_{\gamma f, \max }(z) / A \kappa$.

This equation gives the results shown in Figure 3, plotted as $E^{2} \frac{d F}{d E}$ (the "energy flux") vs. $E$, for the parameter values $\kappa=4 \times 10^{-11}, \eta=1.0, \zeta=0.03, A=5, \Delta \alpha_{q}=0.20$, and $\Delta \alpha_{f}=$ -0.05 . Both the amplitude and shape of the calculated energy flux $E^{2} \frac{d F}{d E}$ matches that of the data, with the exception of the two end data points, which have large systematic uncertainties (not shown) (P. Sreekumar, personal communication, 1995). We note in particular the role of the spectral index shift between the quiescent and flare state populations: if Eq.10 were to be integrated over $\alpha$ using just EGRET's observed spectral index distribution, the minimum in $E^{2} \frac{d F}{d E}$ would displaced to a much lower energy, in contradiction to the data.

Thus we find that in order to match the observed spectral shape of the EGRB, we must invoke a hardening of the flare-state spectra with respect to the quiescent-state spectra, a feature which is already suggested by EGRET observation (Figure 1). In addition, we find that the entire EGRB can be explained as being due to the $\gamma$-ray emissions of unresolved blazars.

\section{Discussion}

We find that those unresolved blazars which make up the $\gamma$-ray background are primarily in the quiescent state, and therefore have a mixture of spectral indices shifted somewhat to the steep side (higher $\alpha$ ) than those which make up the set of observed blazars. This is because the observed blazars contain a higher mixture of flaring sources than those which make up the background, owing to the high EGRET detector threshold and the shape of the high end of the GLF (Figure 2). Thus the EGRET blazar spectral index distribution will be intermediate between that of the flare and quiescent populations, with the mean quiescent-state index being $\Delta \alpha_{q}=0.20$ higher than the observed EGRET distribution, and 


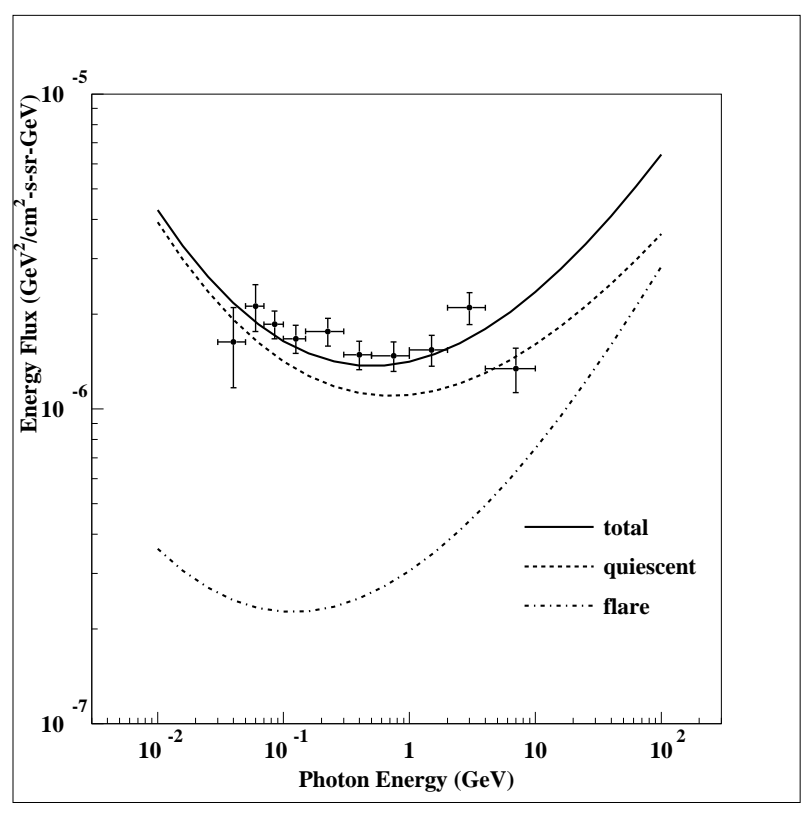

Fig. 3.- The predicted background spectra from summing unresolved blazars compared with the preliminary EGRET data on the cosmic $\gamma$-ray background. The lower, middle, and upper curves show the contribution to the EGRB from flaring sources, from quiescent sources, and the their total. The curves shown are for the parameters indicated in the text, which are the same as those used to generate Figure 2.

the mean flare-state index being $\left|\Delta \alpha_{f}\right|=0.05$ lower than the EGRET distribution. The difference in mean spectral index between the flare and quiescent states, 0.25 , is consistent with the trend indicated in Figure 1. In order to match the concavity of the observed EGRB spectrum, we require a spectral index distribution that is softer than that seen by EGRET, a condition that is met by this two-population model. We note that a general feature of models which posit that the EGRB is due solely to blazars is that the predicted EGRB spectra is concave and not a simple power law. This is due to the EGRB being a superposition of blazar power-law spectra of different spectral indices, where the softer components dominate at lower energies, and harder components dominate at the higher energies.

The calculated EGRB spectrum in Figure 3 is shown down to an energy of $10 \mathrm{MeV}$. Of the few EGRET sources which have been detected by OSSE and COMPTEL below this energy, 7 by COMPTEL and 5 by OSSE (McNaron-Brown et al., 1995 and references therein), almost all show a flattening below $\sim 10 \mathrm{MeV}$. There are not enough sources detected at the lower $\gamma$-ray energies to calculate a background, although it is reasonable to assume that the background from unresolved blazars should also flatten below $10 \mathrm{MeV}$. Note also that the calculated energy flux of the EGRB increases by more than a factor of 2 as one goes from 
$30 \mathrm{MeV}$ down to $10 \mathrm{MeV}$ energy. A preliminary analysis of COMPTEL data (Kappadath $e$ e al., 1995) gives a value for the GRB at $10 \mathrm{MeV}$ which is more than a factor of 2 below our results. It is possible that the discrepancy here may be due to an oversubtraction of detector backgrounds in obtaining the residual GRB from the COMPTEL data, this analysis also having given much lower values than those obtained by APOLLO 15 (Trombka et al., 1977) at lower energies.

Finally, we discuss the parameter values chosen to match the EGRET data. A single flaring factor of $A=5$ is a gross simplification to what is obviously a continuous distribution of flaring factors, and is perhaps as large a value as one should take in this model, although a large $A$ helps distinguish the separate roles of the two blazar states. A surprising result of this calculation is that we were driven to a large value of $\eta$, which is the ratio of solid angles of the beamed $\gamma$-ray emission to the beamed radio emission; in fact, our optimal value is unity. This contrasts with the results of our earlier work (Salamon and Stecker, 1994) and the model of Dermer (1995), where the emitted $\gamma$-rays are emitted into a much smaller solid angle around the jet axis compared to the beamed radio emission. This smaller $\gamma$-ray beaming angle was invoked in our earlier model to explain geometrically why fewer blazars are seen in $\gamma$-rays than in the radio. However, in the present model, the reason fewer $\gamma$-ray blazars are seen is because the ratio $\kappa$ between the $\gamma$-ray and radio luminosities is lower.

The relationship between the parameters $\eta$ and $\kappa$ can be seen in Eq.7. To match the observed number of sources per logarithmic bin of flux, the quantity $\eta \kappa^{\Gamma}$ is fixed, where $\Gamma$ is the local power law of the radio luminosity distribution, $\rho_{r}\left(P_{r}\right) \propto P_{r}^{-\Gamma}$. When we include the effect of flare amplification, $\kappa$ is lowered relative to our earlier model; thus $\eta$ becomes larger than in our earlier model. Owing to the fact that we have introduced two states of blazar activity, we have now have the freedom to raise $\eta$ so as to match the amplitude of the EGRB, while simultaneously respecting the observed number of sources. An indication that this added degree of freedom is not totally ad hoc is seen by the fact that we require a difference in the mean spectral indices of our two blazar populations which is consistent with that suggested by EGRET observations.

Our conclusion then is that both the amplitude and shape of the extragalactic $\gamma$-ray background spectrum as measured by EGRET can be explained as being due solely to the $\gamma$-ray emissions of unresolved blazars, if one assumes a simple relationship between the $\gamma$-ray and radio luminosities of these objects. A critical feature of this simple model is that at a given time, a blazar is in one of two states: a quiescent state or a flare state.

We thank P. Sreekumar for many helpful conversations and criticisms of the manuscript. 


\section{REFERENCES}

Becker, et al., 1991, Astrophys. J. Suppl. 75, 1.

Chiang, J., C.E. Fichtel, C. von Montigny, P.L.Nolan and V. Petrosian, 1995, Astrophys. J. $452,156$.

Dermer, C.D. and Gehrels, N., 1995, Astrophys. J. 447, 103.

Dermer, C.D., 1995, Astrophys. J. 446, L63.

Dunlop, J.S. and Peacock, J.A. 1990, Mon. Not. R. Astron. Soc.247, 19.

Fichtel, C.E., 1995, Proc 3rd Compton Observatory Symposium, Astron Astrophys. Suppl., in press.

Fichtel, C.E., Simpson, G.A. and Thompson, D.J. 1978, Astrophys. J. , 222, 833.

Jang, M. and H.R. Miller 1995, Astrophys. J. 452, 582.

Kappadath, et al. 1995, in Proc. 24th Int. Cosmic Ray Conf., Rome 2, 230.

Kniffen, D.A., et al., 1993, Astrophys. J. 411, 133.

Kühr, H., et al., 1981, Astron. Astrophys. Suppl. 45, 367.

Ledden and O'Dell, 1985, Astron. J. 298, 630.

McNaron-Brown, K., et al. , 1995, Astrophys. J. 451, 575.

Mukherjee, R., et al., 1995 Astrophys. J. 441, L61.

Mukherjee, R., et al. , 1995, submitted to Astrophys. J. .

Padovani, P. Ghisellini, G. Fabian, A.C. and Celotti, A. 1993, Mon. Not. Royal Astron. Soc., 260, L21.

Reich, W. et al., 1993 Astron. Ap. 273, 65.

Salamon, M.H. and F.W.Stecker, 1994, Astrophys. J. 430, L21.

Sreekumar, P., et al. 1995, in preparation.

Stecker, F.W. 1989, Nucl. Phys. B (proc. Suppl), 10B, 93.

Stecker, F.W. De Jager, O.C. and Salamon, M.H. 1992, Astrophys. J. 390, L49. 
Stecker, F.W., Salamon, M.H. and Malkan, 1993, Ap.J. Letters, 410, L71.

Thompson, D.J. and Fichtel, C.E., 1982, Astron. Ap. 109, 352.

Thompson, D.J., et al., 1995, Astrophys. J. Suppl, in press.

Trombka, J.I., et al., 1977, Astrophys. J. 212, 925.

Valtaoja, E. and Teräsranta, H. 1995, Astron. and Ap. 297, L13.

von Montigny, et al., 1995, Astrophys. J. 440, 525.

Wagner, S.J. and A. Witzel 1995, Ann. Rev. Astron. Ap. 33, 163.

Wall, J.V. and Peacock, J.A. 1985, MNRAS, 216, 173. 